\title{
Large gastrointestinal stromal tumour (GIST) in a young female
}

\author{
R. Thayaparren, P. Jeepara \\ University Surgical Unit, Teaching hospital Batticaloa, Sri Lanka
}

\begin{abstract}
Key words: Gastrointestinal stromal tumour; intestitial cells of cajal; c-kit (tyrosine kinase inhibitor) protooncogene
\end{abstract}

\section{Introduction}

Gastrointestinal stromal tumours (GIST) are rare, accounting for less than $1 \%$ of primary neoplasms of the gastrointestinal tract. GIST is defined as immunohistochemically KIT - positive and a KIT signalling primary mesenchymal tumour of the gastrointestinal tract (1). The peak incidence of these tumours are in those of age around 60 years (2) and the tumours can range from small benign tumours to malignant ones. This patient was diagnosed at the age of only 23 years, making it a rare presentation.

\section{Case report}

A 23 year old previously healthy female presented with a history of abdominal distention and loss of appetite for one month with fever and generalized body swelling for the last three days. On physical examination, she was found to be pale and there was a non-tender large intraabdominal epigastric mass extending down to the umbilicus. Investigations revealed anaemia (haemoglobin of $7.1 \mathrm{~g} / \mathrm{dl}$ ) and hypoproteinaemia (total protein of $4.9 \mathrm{~g} / \mathrm{dl}$, albumin of $2.4 \mathrm{~g} / \mathrm{dl}$ ). An ultrasound scan of the abdomen showed a large solid mass lesion seen in the upper abdomen. The contrast enhanced CT (CECT) scan of the abdomen and pelvis revealed a large intraperitoneal mass (measuring $9.9 \mathrm{~cm} \times 13.7 \mathrm{~cm} \times 15$ $\mathrm{cm}$ ) without obvious vascular infiltration. The mass was compressing a part of the stomach and it was suggested to be an intraperitoneal sarcoma. Upper gastrointestinal endoscopy (OGD) revealed two areas of ulceration at the lesser curvature of the stomach. On exploration, there was a solid mass touching the anterior abdominal Correspondence: P. Jeepara E-mail: jeepara@yahoo.com wall and arising from the lesser curvature of the stomach, extending to the first part of the duodenum and it was adherent to the capsule of the left lobe of the liver, the transverse mesocolon and the head of the pancreas. A wide local excision was done in the form of a subtotal gastrectomy and partial duodenectomy after freeing the adhesions. A part of the mesocolon also had to be removed. Reconstruction was in the form of the Hofmeister modification of Billroth-II. The postoperative period was uneventful. The patient was discharged with a plan for further follow up by the oncology unit.

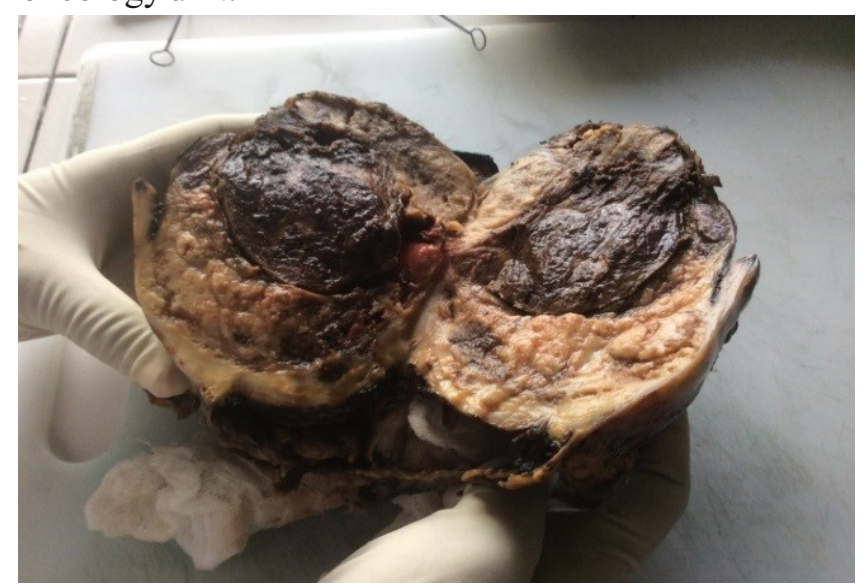

Figure 1. Excised tumour cut section for histology

Microscopically, a mixed spindle and epithelioid cell neoplasm within the distal stomach and proximal duodenal wall was found with a mucosal resection margin of $9 \mathrm{~mm}$ and $15 \mathrm{~mm}$. These cells had marked focal nuclear pleomorphism, hyperchromasia and mitosis at $<5 / 50 \mathrm{HPF}$. The lesion involved the submucosal layer, muscle layer and the sub-serosa of the stomach and proximal duodenal wall. Immunohistochemistry staining found that the cells were CD117 and SMA positive, whilst S100 and CK17 negative. The appearances are keeping in with a gastrointestinal stromal tumour of mixed epitheloid and spindle cell type. 


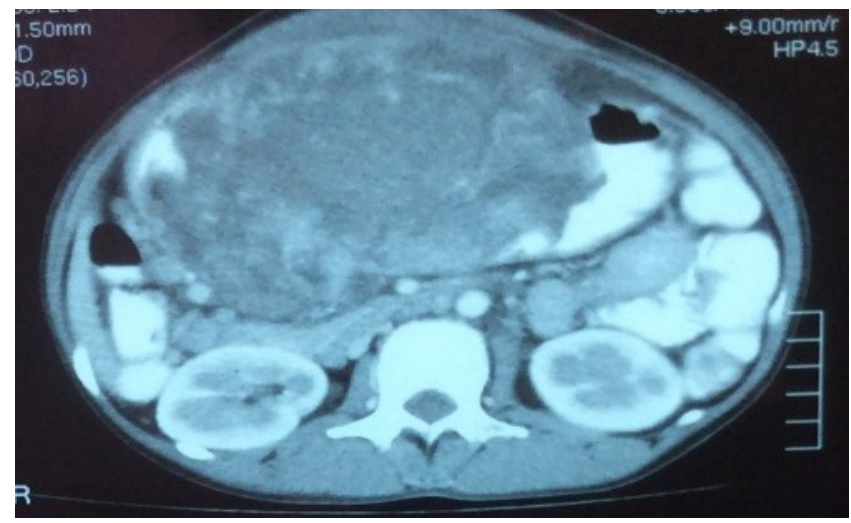

Figure 2. CT scan of the abdomen showing a large tumour

\section{Discussion}

The GISTs are the commonest primary mesenchymal tumour of the gastrointestinal tract and are thought to originate from the interstitial cell of Cajal. GISTs usually affect middle aged and older patients; they are rare before the age of 40 years. GIST incidence is equal in both sexes with a slight male predominance (3). Most commonly it originates in the stomach $(60 \%)$ followed by small intestine $(25 \%)$, the colon and rectum $(5 \%)$ and rarely even in at extra-intestinal sites. This case is particularly unusual because of the extremely large tumour size of $9.9 \mathrm{~cm} \times 13.7 \mathrm{~cm} \times 15 \mathrm{~cm}$ in a 23 year old female. Other authors have reported a mean GIST size of $6-10.6 \mathrm{~cm}$ (7). The clinical presentation is nonspecific; she had a palpable mass, weight loss and anaemia. About $20 \%$ of GISTs are asymptomatic, so the clinical diagnosis of GIST is based on a high index of suspicion. Pre-operative imaging modalities like CECT abdomen and OGD aids in diagnosis. The large lesions show cystic degeneration with central necrosis and ulceration of the overlying mucosa. Microscopically most GISTs consist of a uniform population of spindle cell $(70 \%)$ and epitheloid type $(20 \%)$, which typically arise in the stomach. The remaining $10 \%$ consist of a mixture of these two morphologies, as in this case (4).They exhibit a spectrum of behaviour from benign to malignant lesions. The incidence of malignancy can be predicted based on the size, site and mitotic count. The easiest applicable morphological criteria to predict tumour behaviour is the size.

Gastric tumours more than $10 \mathrm{~cm}$ are probably malignant. Gastric tumours of $\leq 5 \mathrm{~cm}$ in size and with a mitotic count of $\leq 5 / 50 \mathrm{HPF}$ are probably benign. Other prognostic variables are high cellularity, coagulative tumour necrosis, pleomorphism, high s-phase fraction and DNA aneuploidy. There were no soft tissue metastasis in this case. Metastasis beyond the abdomen especially to the bone and lungs is uncommon. The similarities of GISTs with a histological picture of gastrointestinal leiomyosarcoma or a poorly differentiated carcinoma may cause a diagnostic dilemma. The immunohistochemical assay for CD117 antigen is the mainstay for definite diagnosis in $95 \%$ of GISTs. Other markers which may be positive include CD34 (70\%) and smooth muscle actin (SMA) 40\%. CD117 positive GISTs should be considered for molecular analysis for the tyrosine kinase inhibitor or platelet derive growth factor receptor (PDGFR) alpha analysis. This is important for the use of the therapeutic tyrosine kinase inhibitor Imatinib mesylate.

The standard treatment of a localized resectable GIST is surgery. Radio therapy is ineffective. The GISTs are surgically managed on the lines of soft tissue sarcomata (STS). The success of imatinib in controlling locally advanced and metastatic GIST has led to the interest in the neoadjuvant and adjuvant use of drugs. The natural history of malignant GISTs is a protracted course with recurrences and metastasis spanning over years, even up to 10 - 20 years (5). Even among low risk GISTs recurrences have been reported up to 20 years after treatment (2). The predictive factors for recurrence are tumour size $>5 \mathrm{~cm}$.

\section{References}

1. Mieltinenetal M, "Evaluation of malignancy and prognosis of GIST; a review", Human path; 2002; 33(5): 478 - 83.

2. Corlessetal CL, "The biology of gastrointestinal stromal tumours “, JCO 2004; 22(18): 3813-25.

3. Mieltinen M. Lasota J, gastrointestinal stromal tumors definition, clinical, histological, Immunohistochemical and molecular genetic features and differential diagnosis. Virchaws arch 2001; 438; $1-12$.

4. Mieltinen M, Majidi M, Lasota J, 2002. Pathology and diagnostic criteria of gastrointestinal stromal Tumors (GISTs): a review. Eur J cancer 38: 539-51.

5. Nilsson B, Bumming P, Meis - Kindblom JM, et al. 2005. Gastrointestinal stromal tumors: the Incidence,prevalence, clinical course and prognostication in the preimatinibmesylate era - a Population - based study in western Sweden. Cancer, 103: 821 - 9.

6. Bauer S, Hartmann JT, De Wit M,etal2005. resection of residual disease in patients with metastatic gastrointestinal stromal tumours responding to treatment with imatinib. int J cancer 117;316-25.

7. Patil S, Jain S, Kaza R C. et al. Giant gastrointestinal stromal tumor presenting as a palpable abdominal mass: an unusual presentation. ISRN Surg. 2011;2011:894829. 


\section{Key Points:}

- GISTs are the commonest primary mesanchymal tumours of the gastrointestinal tract.

- The clinical presentation is non-specific, often asymptomatic and has a spectrum of behavior ranging from benign to malignant.

- Histopathology \& IHC assay yields the definitive diagnosis.

- Standard treatment is local resection with neo-adjuvant and adjuvant biotherapy.

\section{Answers to images in surgery (Page 31)}

1. Chilaiditi sign. (Segment of bowel interposed between diaphragm and the liver).

2. The normal upper abdominal anatomy prevents hepatodiaphragmatic interposition of bowel in a normal person. Any condition that alters the hepatodiaphragmatic recess results in the above findings. These conditions include decreased liver volume, weakness or division of the suspensory ligaments of the liver and ascites or high intraabdominal fat content. In addition, patients with megacolon or a long, redundant colon with a long, narrow mesentery are at increased risk of developing the condition (congenital anomalies).

Discussion - Chilaiditi sign is an incidental radiologic finding with reported incidence of $0.025 \%$ to $0.28 \%$ in the general population and an important imitator of free air under the diaphragm in symptomatic patients. This entity is termed Chilaiditi syndrome when associated with vague abdominal pain, distension, vomiting, anorexia and constipation. Lateral decubitus films are helpful in differentiation of pneumoperitoneum from hepatodiaphragmatic interposition since unlike pneumoperitoneum, the "air" will remain in the hepatodiaphragmatic recess.

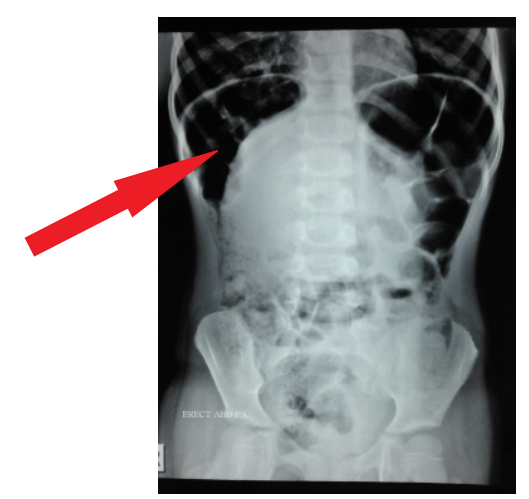

Figure 1.At presentation

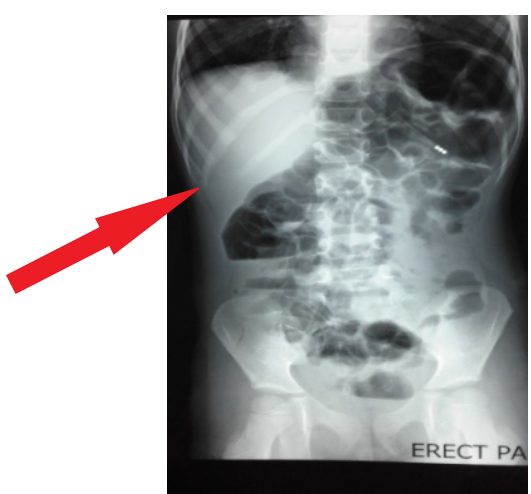

Figure 2. Following conservative management after 48 hours

\section{References}

1. Wei- hong weng, Da-ren lliu, Cheng-cheng feng, Ri-shengque. Colonic interposition between the liver and left diaphragm management of Chilaiditi syndrome: A case report and literature review. Oncol Lett. 2014 May; 7(5): 1657-1660.doi: 10.3892/ol.2014.1903.

2. Risaliti A, De Anna D, Terrosu G, Uzzau A, Carcoforo P, Bresadola F. Chilaiditi's syndrome as a surgical and nonsurgical problem. Surg Gynecol Obstet. 1993 Jan;176(1): 55-8. 